\title{
Management of asthma in patients supervised by primary care physicians or by specialists
}

\author{
L. Laforest*, E. Van Ganse*, G. Devouassoux*, S. Chretin*, L. Osman ${ }^{\top}$, G. Bauguil', \\ Y. Pacheco ${ }^{\#}$ and G. Chamba
}

ABSTRACT: French asthma patients may be supervised by general practitioners (GPs) and/or specialists. Therefore, this study examined asthma management in patients exclusively supervised by specialists (SPE), GPs, (GP) and both (GP+SPE group), and compared the findings.

Asthma patients were consecutively recruited in 348 pharmacies. Each patient completed a questionnaire providing data on personal characteristics, asthma management, perception of disease and asthma supervision. Asthma control was measured using the Asthma Control Test. Questionnaires were linked to computerised records of medications which had been dispensed before inclusion in the study.

From the 1,256 patients (mean age $=36.1 \mathrm{yrs}, \mathbf{5 4 . 3} \%$ females), $11.4,36.6$, and $\mathbf{5 2 . 0} \%$ were placed in the SPE, GP, and GP+SPE groups, respectively. During the previous 4 weeks, most patients in the SPE group were properly controlled (52.2 versus 26.4 and $21.5 \%$ in GP and GP+SPE groups, respectively). The SPE group made more use of fixed combinations of long-acting beta agonist and inhaled corticosteroid, while receiving less short-acting beta agonists, antitussives and antibiotics.

Striking differences in symptoms and asthma management were observed according to the type of asthma supervision. The current results strongly support the need to improve the management of asthma in primary care, and the coordination of care between general practitioners and specialists.

KEYWORDS: Asthma, control, primary care, supervision

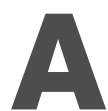
sthma is a major public health problem, given the steady increase of its incidence during recent years and the resulting medical resource utilisation [1, 2]. In addition, in spite of the appearance of new efficient controller therapy $[3,4]$, a substantial proportion of asthma patients remain in inadequate control of their asthma [5]. One reason for this poor control is poor adherence to asthma-controller therapy $[6,7]$.

In France, asthma patients are free to choose to be supervised for their disease by a general practitioner (GP), by a specialist, or by both. Comparison of asthma patients according to their asthma supervision has undergone little investigation. Such comparison may include patients' baseline characteristics, asthma symptoms, management of the disease or a patient's perception of asthma. In particular, it would be of interest to know if patients, supervised by specialists have better controlled asthma than those who are followed in primary care, and whether dispensed antiasthma medications differ according to the type of asthma supervision.

The aim of the present study was to compare patients' characteristics and management according to whether they were managed within primary care or by specialists. The study's specific interest was the level of asthma control achieved by patients. Medical resource utilisation, use of medications, and patient perceptions, regarding asthma, were also compared according to supervision.

\section{METHODS}

\section{Study design and study population}

The design of the present study has been presented in a previous article [8]. A retrospective observational study was carried out in nine cities in France (Lyon, Montpellier, Caen, Nancy, Amiens, Dijon, Angers, Limoges, Besançon). Asthma patients were consecutively

\section{AFFILIATIONS}

*Pharmacoepidemiology Unit,

${ }^{\#}$ Respiratory Medicine, CHU-Lyon, and

${ }^{+}$Faculty of Pharmacy, ClaudeBernard University, Lyon, France. -University of Aberdeen, Aberdeen, UK

CORRESPONDENCE

E. Van Ganse

Unité de Pharmacoépidémiologie EA3091

Ste Eugénie (5F) Centre Hospitalier Lyon-Sud F-69495 Pierre-Bénite Cedex

France

Fax: 33472666444

E-mail: eric.van-ganse@chu-lyon.fr

Received:

March 252005

Accepted after revision:

August 052005

SUPPORT STATEMENT

This study was supported by

GlaxoSmithKline France. 
recruited in pharmacies (between November 2003 and January 2004). Community pharmacists invited asthma patients aged 18-50 yrs, who were regular users of the pharmacy, to participate in the study. Patients taking a prescription of an antiasthma medication (R03, Anatomical Therapeutic Chemical classification system) were included. The protocol had been sent for approval, as requested by local regulations, to the National Committee of Informatics and Liberty, a French regulatory body.

\section{Data collected}

Patients who met the inclusion criteria and who agreed to participate in the study completed a questionnaire assessing their main baseline characteristics, asthma symptoms and asthma-related medical resource utilisation (except medications). Patients also categorised how they perceived the impact of their disease on their life. Lastly, they had a peak flow measurement taken at the pharmacy during their inclusion visit. The percentages of theoretical values were computed based on Quanjer formula.

\section{Dispensed medications and other asthma-related medical resource utilisation}

Antiasthma medications dispensed to patients before inclusion were available through computerised pharmacy records. Controller medication classes included: inhaled corticosteroids (ICS), long-acting beta agonists (LABA), LABA-ICS fixed combinations, oral xanthine, and leucotriene receptor antagonists. Reliever therapy included: short-acting beta agonists (SABA) and anticholinergic medications. The dispensing of oral corticosteroids, antibiotics, expectorants and antitussives were also recorded. For each medication class, the number of units dispensed monthly was available. Patients reported in questionnaires the average frequency of their visits to GPs or respiratory physicians and their asthma-related hospital contacts during the past 12 months.

\section{Level of asthma control}

The level of asthma control was measured with the Asthma Control Test [9]. The questionnaire covers frequency of symptoms, impairment of daily activities and the need for rescue medication during the past 4 weeks. The French version has been validated. Patients also made a self-assessment of their own level of control (table 1). The scoring method for control was derived from the sum of counts option [9]. Each question included five items (a-e), classified according to decreasing level of asthma control. The last three items (c-e) corresponded to an inadequate level of control. When the patients did not check any of these items for any question, their asthma was considered to be properly controlled. When items c-e were checked for one or two questions, patients were considered as "moderately uncontrolled". In all other cases they were classified as poorly controlled.

\section{Definition of asthma supervision}

In the questionnaire, patients reported the average frequency of their asthma-related medical visits to GPs and to respiratory physicians. Three groups of patients were defined: patients who were only supervised by specialists (SPE group), those only supervised in primary care (GP group), and those who visited both GPs and specialists (GP+SPE group). The present analyses were focused on patients with documented dispensation records and who mentioned their asthma-related visits to GPs and to respiratory physicians.

\section{Analyses}

The three groups were compared for 1) patients' demographic data, 2) asthma-related characteristics (level of control, symptoms, peak-flow measures), 3) medical resource use and 4) perception of the disease by the patients and their need for information. To allow for the potential confounding influence of asthma severity, the comparisons of asthma control between groups were also performed, after stratifying on the dispensation of oral corticosteroids and on peak flow measures. Statistical differences between the three groups were tested with chi-squared test for qualitative variables, and with Kruskal-Wallis test for quantitative variables. Medication resource use was studied in patients who had at least 6 months of dispensed medication records available.

\section{RESULTS}

Of the total 1,559 patients recruited from the 348 pharmacies, 1,256 patients had dispensing records available and gave data on their average frequency of visits to GPs and specialists. Of these $460(36.6 \%)$ only visited a GP, $653(52.0 \%)$ visited both a GP and a specialist, and $143(11.4 \%)$ were exclusively supervised by a specialist. In combination, a total of 1,199 patients had at least 6 months of computerised records available (fig. 1).

\section{Patients' baseline characteristics}

There were significantly fewer smokers in patients supervised by specialists. Patients in the SPE group were younger than in the other two groups (table 2). The three patient groups did not differ with respect to sex and body mass index.

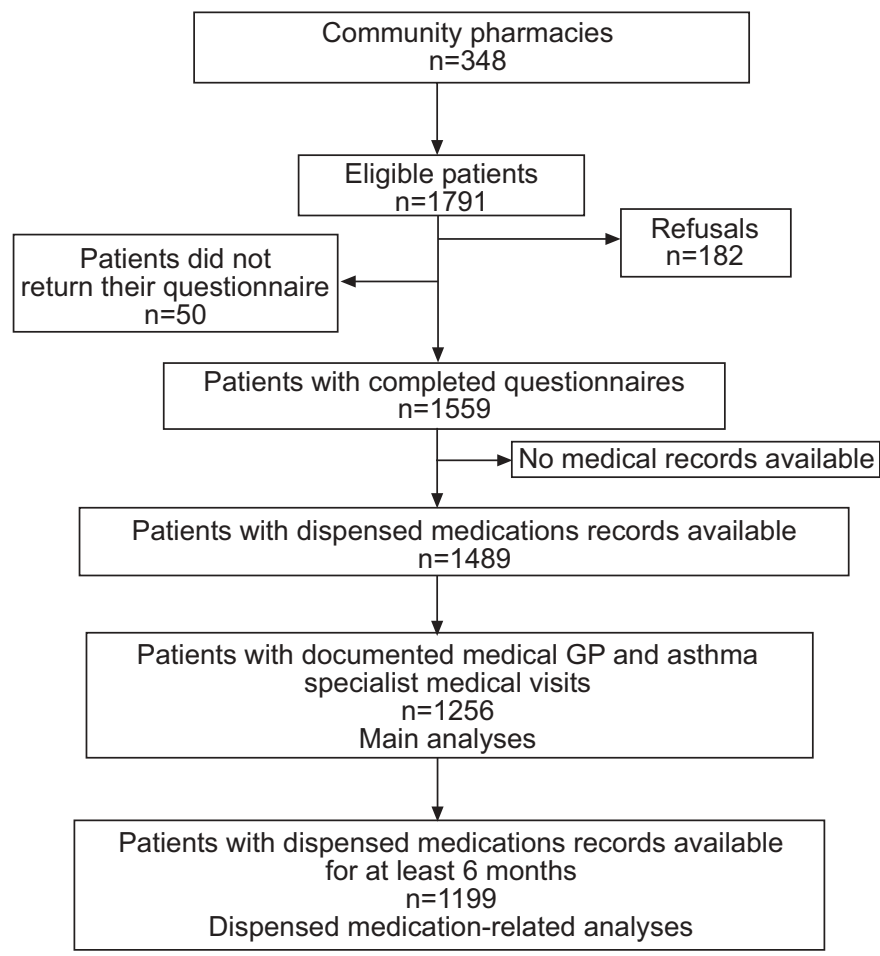

FIGURE 1. Definition of the study population. GP: general practitioner. 
TABLE 1 Comparison of asthma control between groups

GP

Group

Overall

p-value

GP+SPE

1. During the past 4 weeks, for how much of the time did your asthma prevent you from getting as much done as normal at work or at home?
a) None of the time
b) A little of the time
c) Some of the time
d) Most of the time
e) All of the time

2. During the past 4 weeks, how often have you had shortness of breath?
a) Not at all
b) Once or twice a week
c) 3-6 times a week
d) Once a day
e) More than once a day

3. During the past 4 weeks, how often did your asthma symptoms (wheezing, coughing, shortness of breath, chest tightness or pain) wake you up at night or earlier than usual in the morning?
a) Not at all
b) Once or twice
c) Once a week
d) 2-3 nights a week
e) $\geqslant 4$ nights a week

4. During the past 4 weeks, how often have you used your rescue inhaler or nebulizer medication (such as salbuterol)?
a) Not at all
b) Once a week or less
c) A few times a week
d) 1 or 2 times per day
e) $\geqslant 3$ times per day

5. How would you rate your asthma control during the past 4 weeks?
a) Completely controlled
b) Well controlled
c) Somewhat controlled
d) Poorly controlled
e) Not at all controlled

Level of asthma control

$\begin{array}{ll}\text { Correct } & 117(26.4) \\ \text { Moderate } & 178(40.2) \\ \text { Poor } & 148(33.4)\end{array}$

$\begin{array}{cc}177(38.7) & 199(30.5) \\ 126(7.6) & 130(19.9) \\ 127(27.8) & 251(38.4) \\ 22(4.8) & 53(8.1) \\ 5(1.1) & 20(3.1) \\ & \\ 79(17.4) & 111(17.2) \\ 194(42.7) & 238(36.9) \\ 51(11.2) & 72(11.2) \\ 67(14.8) & 74(11.5) \\ 63(13.9) & 150(23.3)\end{array}$

$163(35.7)$

$138(30.3)$

61 (13.4)

$54(11.8)$

$40(8.8)$

$96(21.1)$
$107(23.5)$
$105(23.1)$
$94(20.7)$
$53(11.6)$
$102(22.3)$
$237(51.7)$
74 (16.2)
$24(5.2)$
$21(4.6)$
$148(33.4)$

151 (23.6)

$135(21.1)$

$150(23.5)$

$120(18.8)$

$83(13.0)$

$214(33.2)$
$194(30.1)$
$75(11.6)$
$88(13.6)$
$74(11.5)$

$89(62.7)$

28 (19.7)

$11(7.7)$

$5(3.5)$

$9(6.3)$

$\begin{array}{ll}70(50.0) & 317(25.7) \\ 40(28.6) & 282(22.9) \\ 17(12.1) & 272(22.0) \\ 7(5.0) & 221(17.9) \\ 6(4.3) & 142(11.5)\end{array}$

$466(37.5)$
$360(29.0)$
$147(11.8)$
$147(11.8)$
$123(9.9)$
0.000

0.000

240 (19.3)

489 (39.4)

$133(10.7)$

151 (12.2)

228 (18.4)
0.000

0.000

0.000

$\begin{array}{cc}58(40.6) & 295(23.6) \\ 62(43.4) & 651(52.2) \\ 15(10.5) & 204(16.3) \\ 7(4.9) & 60(4.8) \\ 1(0.7) & 38(3.0) \\ 72(52.2) & 323(26.8) \\ 43(31.2) & 454(37.7) \\ 23(16.7) & 426(35.4)\end{array}$

Data are presented as n (\%) unless otherwise specified. GP: general practitioner; SPE: specialist. Correct asthma control: items c, $d$ or e were never checked for any of the five questions; moderately uncontrolled asthma: items c, d or e were checked for one or two questions; poor asthma control: items c, d or e were checked for at least three questions.

\section{Asthma characteristics}

Peak-flow meter measurements were significantly higher in patients exclusively supervised by specialists. Diurnal and nocturnal asthma attacks were significantly less frequent in this group (table 2). Twice as many patients in the SPE group had satisfactory control of their asthma than in the GP and the $\mathrm{GP}+\mathrm{SPE}$ groups. In contrast, $>40 \%$ of patients in the $\mathrm{GP}+\mathrm{SPE}$ group and $\sim 33 \%$ of patients in the GP group had 


\section{TABLE 2 Comparison of patient characteristics}

\begin{tabular}{|c|c|c|c|c|c|}
\hline & \multicolumn{3}{|c|}{ Group } & \multirow[t]{2}{*}{ Overall } & \multirow[t]{2}{*}{ p-value } \\
\hline & GP & GP+SPE & SPE & & \\
\hline Age mean \pm SD & $35.3 \pm 9.5$ & $36.9 \pm 10.0$ & $34.7 \pm 10.0$ & $36.1 \pm 9.9$ & 0.003 \\
\hline Sex female & $243(52.9)$ & $369(56.7)$ & $69(48.3)$ & $681(54.3)$ & 0.140 \\
\hline BMI & & & & & 0.599 \\
\hline $25-30 \%$ & $79(18.5)$ & $120(20.3)$ & $22(17.1)$ & $221(19.2)$ & \\
\hline$>30 \%$ & $55(12.9)$ & $87(14.7)$ & $13(10.1)$ & $155(13.5)$ & \\
\hline Smoking & & & & & 0.000 \\
\hline Nonsmokers & $267(58.6)$ & $472(73.0)$ & $120(85.1)$ & $859(69.1)$ & \\
\hline $1-9$ cigarettes $\cdot$ day $^{-1}$ & 79 (17.3) & $84(13.0)$ & $11(7.8)$ & $174(14.0)$ & \\
\hline$\geqslant 10$ cigarettes $\cdot$ day $^{-1}$ & $110(24.1)$ & $91(14.1)$ & $10(7.1)$ & $211(17.0)$ & \\
\hline $31-60 \%$ & $75(17.4)$ & $103(17.1)$ & $9(6.9)$ & $187(16.1)$ & \\
\hline Frequency of diurnal asthma & & & & & 0.028 \\
\hline Once a week or less & $299(76.3)$ & $396(72.4)$ & $104(86.0)$ & $799(75.4)$ & \\
\hline >once a week & $52(13.3)$ & $92(16.8)$ & $9(7.4)$ & $153(14.4)$ & \\
\hline$\geqslant$ once daily & $41(10.5)$ & $59(10.8)$ & $8(6.6)$ & $108(10.2)$ & \\
\hline Frequency of nocturnal asthma attacks & & & & & 0.000 \\
\hline Never & $231(56.1)$ & $282(50.4)$ & $98(78.4)$ & $611(55.7)$ & \\
\hline$>2$ monthly & $120(29.1)$ & $177(31.7)$ & $20(16.0)$ & $317(28.9)$ & \\
\hline >once a week & $38(9.2)$ & $67(12.0)$ & $3(2.4)$ & $108(9.9)$ & \\
\hline Almost each night & $23(5.6)$ & $33(5.9)$ & $4(3.2)$ & $60(5.5)$ & \\
\hline
\end{tabular}

All data are presented as n (\%) unless otherwise stated. GP: general practitioner; SPE: specialists; BMI: body mass index.

poorly controlled asthma, compared with $17 \%$ in SPE group. Significant statistical differences between groups were observed for each question of the asthma control test (table 1).

When asthma control was compared between groups in patients with no dispensed units of oral corticosteroids, a two-fold higher proportion of controlled patients was still observed in the SPE group: 50.7 versus $27.6 \%$ in the GP group and $24.8 \%$ in the GP+SPE group $(\mathrm{p}<0.0001)$. Similarly, in patients with peak flow meter measures $>80 \%$ of the predicted value, those with correct asthma control were $59.6 \%$ in the SPE group, compared with 32.0 and 27.5 in the GP and GP+SPE groups $(p<0.0001)$. These differences of asthma control persisted between groups in patients with oral corticosteroids, and in those with peak flow meter measures $<80 \%$ of predicted value (data not shown). Lastly, the difference of proportions of properly controlled patients between the three groups remained virtually unchanged when considering only nonsmoker patients $<40$ yrs (51\% in the SPE group, compared with 39 and $27 \%$ in the GP and GP+SPE groups, respectively; $\mathrm{p}=0.006$ ).

\section{Medical resource utilisation and asthma management}

Medical resource utilisation (other than medications) and written action plans in case of asthma attacks

Most patients supervised in primary care (GP and GP+SPE groups) reported visits to GPs four times a year. However, a substantial minority of them had monthly visits, especially in the GP+SPE group (table 3). Visits to specialists occurred essentially twice or three times a year for patients in the SPE group. In the GP+SPE group, the frequency of these visits was annual. Asthma-related hospital admissions were approximately twice as frequent in the GP+SPE group, compared with other groups (table 3).

\section{Dispensing of medications}

Records of dispensed medications were available for 1,199 patients. The average duration of dispensing records was 10.2 months $(\mathrm{SD}=2.6)$. Duration ranged from 3-18 months. There were no significant differences between the three groups in record duration $(\mathrm{p}=0.66)$.

\section{Antiasthma treatments}

Compared with patients of the GP and GP+SPE groups, those followed exclusively by specialists were dispensed significantly more LABA-ICS combinations (table 4). A similar trend was observed for leucotriene antagonist receptors. In contrast, more oral xanthines were dispensed in the GP+SPE group. Dispensing of inhaled corticosteroids without any associated LABA was unusual in the SPE group. Reliever medications, such as SABA, were significantly less observed in dispensing records when patients were exclusively supervised by specialists (table 4). 
Overall changes in therapy occurred significantly more often in the GP+SPE group. Decrease of dosage and withdrawal of medication were more often observed in the SPE group (table 3).

\section{Other treatments}

Oral corticosteroids were more often dispensed in the GP+SPE group than in the other groups. In contrast, patients supervised in primary care (GP or GP+SPE groups) were dispensed significantly more antitussives expectorants and antibiotics (table 4).

\section{Perception of asthma and need for additional information}

Perception of asthma differed according to the type of supervision (table 5). Only one-quarter of patients in the SPE group perceived their asthma as a handicap or a major concern in their lives, compared with nearly $40 \%$ in the GP group and $50 \%$ in the GP+SPE group. Patients exclusively supervised by specialists were significantly more likely to consider that they were adequately informed about their disease. No difference was observed between groups with respect to perceived need for additional information regarding antiasthma treatments. Patients in the GP+SPE group were most likely to search spontaneously for information on their disease.

\section{DISCUSSION}

Characteristics of asthma patients and the management of their disease were compared between patients according to their type of supervision. Patients exclusively followed by specialists (SPE group) had significantly better controlled asthma than other patients and experienced fewer asthma symptoms. They received significantly more LABA-ICS combinations. Conversely, they were less frequently dispensed antibiotics, antitussives or expectorants. Perception of the burden of asthma was less marked in the SPE group than in the other patient groups. Patients followed by specialists were less likely to declare themselves inadequately informed about their disease than their counterparts supervised in primary care.

Only a minority of patients were exclusively supervised by specialists (11.2\%). Most asthma patients in the study were supervised in primary care. The GP+SPE group included patients who visited both GPs and specialists for their asthma (table 3). More than half of the patients in this group had visited a specialist only once in a year. Compared with the group who only visited a GP, the GP+SPE group of patients may have been perceived as having more severe asthma, as suggested by the higher dispensing of oral corticosteroids to this group and a two-fold higher frequency of asthma-related hospital admissions (table 3). The poorest level of control was observed in this group (tables 1 and 2), and patients in this group, more significantly than other patients, perceived adverse consequences of asthma in their lives. The current study's results suggest that there is no real coordination between GPs and specialists. These patients may visit a specialist in case of an emergency context, without any coordinated preventative action or follow-up.

Well controlled patients were twice as numerous in the SPE group as in others. The differences between groups were significant for all components of asthma control (table 1). The current study population may not be representative of the general population of asthma patients, more specifically for severity. This could be problematic for the generalisation of the current findings. However, the difference in asthma control between groups remained significant when the comparisons were stratified according to the use of oral corticosteroids. Similar conclusions were reached when comparisons were

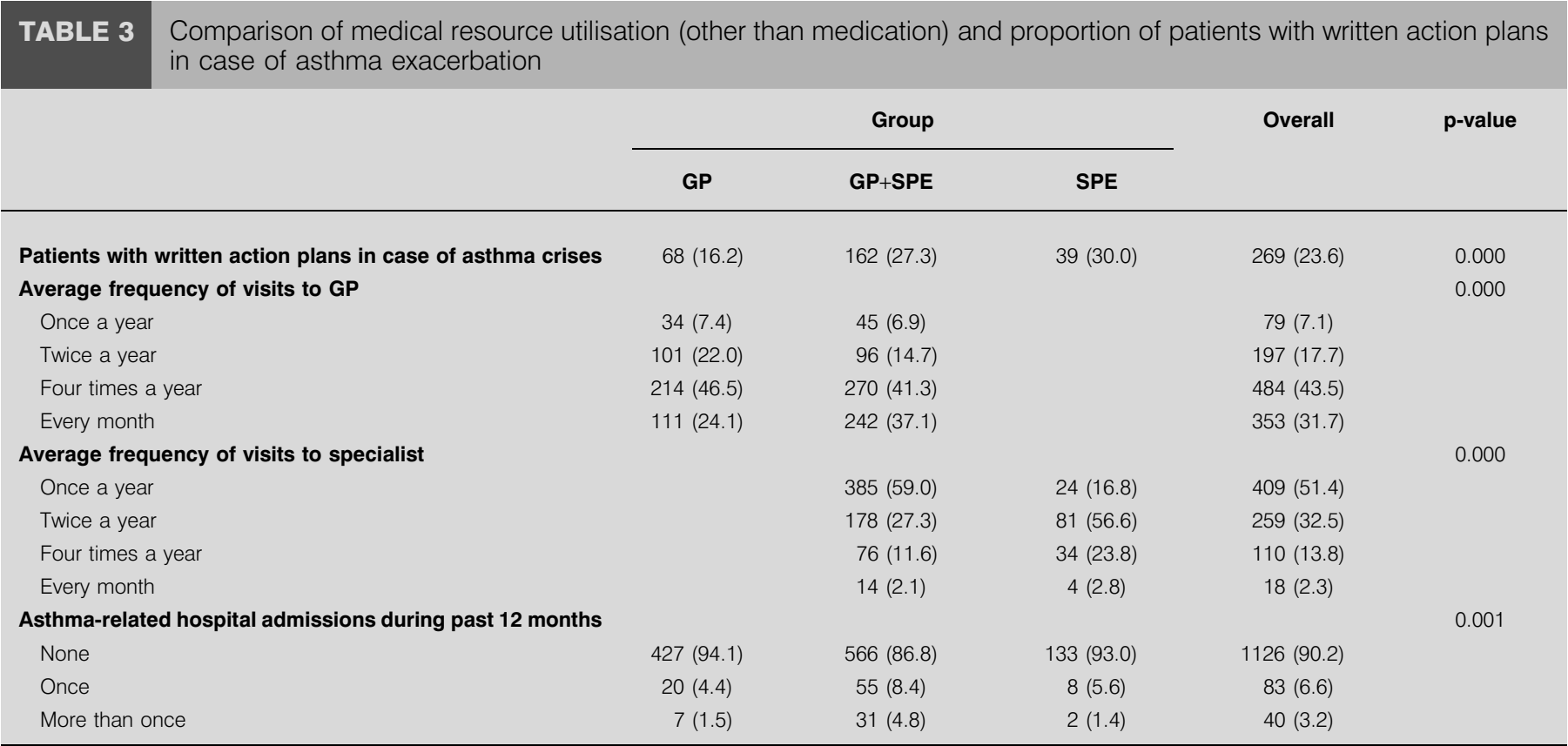


performed after stratifying according to peak-flow meter measurements. These complementary analyses highlight the robustness of the current data, and suggest that the present conclusions are also applicable to less severe patients.

Improved peak-flow measurements were observed in the SPE group and asthma symptoms were also less frequent (table 2). These data confirm the findings of a previous study [10]. In this study, higher rates of symptoms, emergency room visits, lost work days and asthma-related hospital admission have been reported in asthma patients supervised in primary care.

Most patients in the three groups were dispensed anti antiinflammatory medication, which is in accordance with the official Global Initiative for Asthma guidelines [11]. However, patients supervised by respiratory physicians received significantly more LABA-ICS combinations as controller therapy. The beneficial impact on asthma control of this association has been established $[3,4]$. According to the Global Initiative for Asthma guidelines, such a difference in prescription patterns would be in favour of a higher severity of the patients in the SPE group, though these results may be difficult to interpret given the absence of evidence regarding the adherence to the guidelines in daily practice. In contrast, patients in the SPE group were dispensed significantly fewer asthma relievers (SABA). A significantly higher proportion of patients in the SPE group reported a decrease of asthma therapy or withdrawal of any antiasthma medication during the past 12 months, consistent with the lower symptoms observed in this group.

Other medication classes, such as antibiotics, expectorants or antitussives, were more often dispensed in the GP and GP+SPE groups (table 4) than in the SPE group. In a previous study conducted between 1996 and 1998 based on a GP computerised database, a substantial proportion of patients with antibiotic prescriptions were identified during a 12-month study period (57.6\%) [12]. Though direct comparison is difficult between prescription and dispensing data, similar percentages of

TABLE 4 Comparison of dispensed treatments ${ }^{\#}$

\section{Group}

GP + SPE
Overall

SPE p-value
GP

\section{Antiasthma-controller therapy}

ICS

LABA

COMBO LABA-ICS

Oral xanthines

LTRA

\section{Controller regimens}

Isolated ICS

ICS+LABA (separate medications)

COMBO LABA-ICS

COMBO ABA-ICS+ICS

COMBO LABA-ICS+LTRA

Others/none

\section{Antiasthma reliever medications}

SABA

Anticholinergic medication

Anticholinergic medication +SABA

\section{Other medications}

Oral corticosteroids

Antitussives

Expectorants

Antibiotics

Change of therapy during the past 12

months

None

Addition of medication(s)

Suppression of medication(s)

Any increase of dosage

Any decrease of dosage

Total change of therapy
$129(29.5)$

$243(55.5)$

$21(4.8)$

$90(20.5)$

50 (11.4)

$60(13.7)$

$123(28.1)$

22 (5.0)

43 (9.8)

140 (32.0)

323 (73.7)

$4(0.9)$

32 (7.3)

166 (37.9)

$131(29.9)$

$214(48.9)$

254 (58.0)
311 (49.8)

221 (35.4)

373 (59.8)

51 (8.2)

204 (32.7)

48 (7.7)

85 (13.6)

$142(22.8)$

33 (5.3)

81 (13.0)

$235(37.7)$

454 (72.8)

13 (2.1)

61 (9.8)

297 (47.6)

$176(28.2)$

357 (57.2)

397 (63.6)
59 (43.1)

41 (29.9)

97 (70.8)

3 (2.2)

46 (33.6)

4 (2.9)

20 (14.6)

$42(30.7)$

$11(8.0)$

23 (16.8)

37 (27.0)

79 (57.7)

$0(0.0)$

11 (8.0)

52 (38.0)

26 (19.0)

51 (37.2)

60 (43.8)
554 (46.2)

391 (32.6)

713 (59.5)

75 (6.3)

340 (28.4)

102 (8.5)

165 (13.8)

307 (25.6)

66 (5.5)

147 (12.3)

412 (34.4)

856 (71.4)

$17(1.4)$

104 (8.7)

515 (43.0)

333 (27.8)

622 (51.9)

711 (59.3)
0.031

0.097

0.006

0.009

0.000

0.004

61 (42.7)

$30(21.0)$

17 (11.9)

16 (11.2)

27 (18.9)

12 (8.4)
531 (42.3)

416 (33.1)

193 (15.4)

147 (11.7)

$100(8.0)$

$99(7.9)$
0.001

0.356

0.003

0.042

0.000

0.000

Data are presented as $n(\%), n=1,199 .{ }^{*}$ : Presence of medication category in the computerised dispensing record. ICS: inhaled corticosteroids; LABA: Iong-acting beta agonists; COMBO: combination; LTRA: leucotriene receptor antagonists. SABA: short-acting beta agonists; GP: general practitioner; SPE: specialist. 


\begin{tabular}{|c|c|c|c|c|c|}
\hline & \multicolumn{3}{|c|}{ Group } & Overall & p-value \\
\hline $\begin{array}{l}\text { Feels enough informed regarding } \\
\text { asthma }\end{array}$ & $327(71.7)$ & $481(74.9)$ & $120(84.5)$ & $928(74.8)$ & 0.009 \\
\hline $\begin{array}{l}\text { Patients searched spontaneously for } \\
\text { information regarding asthma }\end{array}$ & $167(37.3)$ & $353(55.8)$ & 65 (46.1) & $585(47.9)$ & 0.000 \\
\hline $\begin{array}{l}\text { Asthma considered as a major } \\
\text { concern or a handicap }\end{array}$ & $169(38.1)$ & $293(47.2)$ & 35 (25.2) & 497 (41.3) & 0.000 \\
\hline
\end{tabular}

Data are presented as n (\%). GP: general practitioner; SPE: specialist.

antibiotics were observed in the present study in the GP (58.0\%) and GP+SPE groups (58.0 and 63.6\%, respectively). Expectorants and antitussives were also commonly dispensed (19.9 and $42 \%$ of patients, respectively). It has been reported in former studies that GPs are more likely to prescribe antibiotics in asthma exacerbations [13, 14], while specialists are more likely to increase inhaled corticosteroids [15]. The present study suggests that the use of antibiotics in asthma remains common in primary care despite the appearance of new efficient controller therapies.

Asthma patients supervised in primary care were more likely to be smokers. Compared with other groups, a two-fold lower proportion of heavy smokers $\left(\geqslant 10\right.$ cigarettes $\cdot$ day $\left.^{-1}\right)$ was observed in the SPE group (table 2). Helping patients to give up smoking is a major aim in asthma patients, not only because of the well known detrimental consequences of tobacco on health, but because tobacco may also impair the efficiency of antiasthma anti-inflammatory medications [16]. Only a quarter of patients had written action plans in case of asthma attacks, in spite of their well established beneficial impact [17]. This proportion was markedly lower in the GP group (table 3 ).

Patients in the GP and GP+SPE groups reported a greater impact of asthma in their lives than those exclusively managed by specialists. They were more likely to feel a need for further information regarding their disease (table 5). These results are in line with those observed in previous studies, which reported a better quality of life in asthma patients supervised by specialists [18, 19]. Likewise, satisfaction regarding their asthma management and their relationship with their physician was greater when patients were supervised by a specialist [10]. There was no reason to think that this difference may be due to milder asthma in the SPE group; similar levels of oral corticosteroids were observed in both groups (table 4). In the 763 patients who had 12 months of computerised dispensing records, patients who had bought oral corticosteroids were 40 and $45 \%$ in the GP and SPE groups, respectively. The frequency of asthma-related hospital admission during the past 12 months before inclusion did not differ between the two groups (table 3).
A significant limitation of the study was the absence of information on socioeconomic level. The level of education may influence patients' behaviour with regard to health problems. A statistical association has been reported between patients' education level and quality if life in asthma [19]. It cannot be excluded that some patients with a higher educational level may have decided to have their asthma managed by a specialist. These patients may be more sensitive to preventive behaviours. Further studies would be helpful to confirm these findings

In addition, the current sample of regular users of the pharmacies may not be representative of the overall general population of asthma patients. Only regular customers were recruited in order to maximise the reliability and the comprehensiveness of the computerised dispensing records. The current data clearly indicate that asthma management was not optimal in these selected patients who regularly buy their antiasthma medication. It can be assumed that even worse results may have been observed in a more representative sample of asthma patients.

The number of months of computerised dispensing records available before inclusion varied according to patient. Consequently, the actual dispensing level of patients with shorter records may be underestimated. However, the average duration of computerised recording did not significantly differ across the three groups $(p=0.66)$. Lastly, a sensitivity analysis was conducted in patients with a 12-month dispensing record before inclusion. Results between groups remained virtually unchanged (data not reported).

Patients could obtain their medications in other pharmacies, which would lead to underestimation of their actual level of dispensed medications. To limit this potential bias, only patients who regularly obtained their medications in the pharmacy of recruitment were included in the survey. Furthermore, this bias did not prevent the identification of high levels of dispensing for reliever, rescue medications and other classes, such as antitussives or expectorants (table 4).

The clinical reasons for dispensing drugs, other than as antiasthma medications, were not known. However, in this 
young population, it can be assumed that these treatments may have been essentially dispensed for asthma.

Lastly, the diagnosis of asthma in the patients included in the study was not confirmed by a specialist. Consequently, the current sample may include some chronic obstructive pulmonary disease patients, especially in the GP and GP+SPE groups, which might partly account for the higher proportions of smokers observed in these groups. To limit this potential bias, age limit for inclusion was set at $50 \mathrm{yrs}$. In addition, when sensitivity analyses were conducted in nonsmokers and in younger patients ( $<40 \mathrm{yrs}$ ), differences between the three groups remained virtually unchanged (data not reported). If these biases cannot be formally excluded, the authors perceive that their influence over the current results was limited.

These findings deserve attention, especially before the implementation of a new organisation of the healthcare system in France. Asthma management must improve, and improved coordination between GPs and specialists is needed. Though improved outcomes were observed regarding asthma management in patients exclusively supervised by specialists, asthma control and patients' quality of life could be also improved in this group. Until now, in France, patients could be supervised either in primary care or by a specialist according to their own choice. From now on, if they want to be refunded as before, patients will have to first visit a general practitioner and be referred, by the GP, to a specialist when necessary. A similar study after the implementation of this new organisation would be of interest. It is unclear whether patients will continue to choose to be exclusively followed by specialists when a more limited refund is offered. Likewise, the evolution of asthma management in the GP+SPE group may provide interesting data with respect to the consequences of this new organisation on the quality of care.

Implementation of educational programmes would be useful in improving the management of asthma and patients' asthma control, especially in primary care. The beneficial impact of such educative campaigns has been reported by previous studies [20]. Interestingly, many patients in the GP and GP+SPE groups wanted additional information regarding their disease (table 5). Educational programmes should focus on improving adherence to asthma-controller therapy. Most patients were dispensed anti-inflammatory medications (table 3). However, this does not necessarily mean that these treatments were actually used by the patients. Many recent studies conducted in asthma patients reported a poor adherence to inhaled corticosteroids treatment [6]. Furthermore, a significant relationship has been identified between this inadequate adherence and the occurrence of asthmarelated adverse events, such as hospital admissions [7]. Physicians should be more sensitive to the utility of written action plans for their patients to aid in management of asthma exacerbations.

In conclusion, prior to the implementation of a new organisation of healthcare in France, the current survey highlights the differences in asthma management between patients supervised by specialists and general practitioners. Better coordination between general practitioners and specialist would be helpful to improve asthma management of asthma patients.

\section{ACKNOWLEDGEMENTS}

The authors would like to thank the pharmacists and the patients who agreed to participate in this study.

\section{REFERENCES}

1 Van Ganse E, Laforest L, Pietri G, et al. Persistent asthma: disease control, resource utilisation and direct costs. Eur Respir J 2002; 20: 260-267.

2 Gendo K, Lodewick MJ. Asthma economics: focusing on therapies that improve costly or outcomes. Curr Opin Pulm Med 2005; 11: 43-50.

3 Condemi JJ, Goldstein S, Kalberg C, Yancey S, Emmett A, Rickard K. The addition of salmeterol to fluticasone propionate versus increasing the dose of fluticasone propionate in patients with persistent asthma. Salmeterol Study Group. Ann Allergy Asthma Immunol 1999; 82: 383-389.

4 Kavuru M, Melamed J, Gross G, et al. Salmeterol and fluticasone propionate combined in a new powder inhalation device for the treatment of asthma: a randomized, double-blind, placebo-controlled trial. I Allergy Clin Immunol 2000; 105: 1108-1116.

5 Rabe KF, Adachi M, Lai CK, et al. Worldwide severity and control of asthma in children and adults: the global asthma insights and reality surveys. J Allergy Clin Immunol 2004; 114: 40-47.

6 Krishnan JA, Riekert KA, McCoy JV, et al. Corticosteroid use after hospital discharge among high-risk adults with asthma. Am J Respir Crit Care Med 2004; 15:170: 1281-1285.

7 Williams LK, Pladevall M, Xi H, et al. Relationship between adherence to inhaled corticosteroids and poor outcomes among adults with asthma. J Allergy Clin Immunol 2004 114: 1288-1293.

8 Laforest L, Van Ganse E, Devouassoux G, et al. Quality of asthma care: results from a community pharmacy based survey. Allergy 2005; 60: 1505-1510.

9 Nathan RA, Sorkness CA, Kosinski M, Schatz, et al. Development of the asthma control test: a survey for assessing asthma control. J Allergy Clin Immunol 2004; 113: 59-65.

$10 \mathrm{Wu} \mathrm{AW}$, Young Y, Skinner EA, et al. Quality of care and outcomes of adults with asthma treated by specialists and generalists in managed care. Arch Intern Med 2001; 161: 2554-2560.

11 Global Initiative for Asthma. Global Strategy for Asthma Management and Prevention NHLBI/WHO WORKSHOP report. National Institute of Health, Bestheda, Maryland, 2002.

12 Laforest L, Pacheco Y, Bousquet J, Yin D, Sazonov Kocevar V, Van Ganse E. How appropriate is asthma in general practice. Fundam Clin Pharmacol 2005; 19: 107-115.

13 Kljakovic M, Mahadevan G. General practitioner prescribing of antibiotics for asthma. Br J Gen Pract 1998; 48: 1773-1774.

14 Jones K, Gruffydd-Jones K. Management of acute asthma attacks associated with respiratory tract infection: a postal 
survey of general practitioners in the UK. Respir Med 1996; 90: 419-425.

15 Cicutto LC, Llewellyn-Thomas HA, Geerts WH. The management of asthma: a case-scenario-based survey of family physicians and pulmonary specialists. J Asthma 2000; 37: 235-246.

16 Chalmers GW, Macleod KJ, Little SA, Thomson LJ, McSharry CP, Thomson NC. Influence of cigarette smoking on inhaled corticosteroid treatment in mild asthma. Thorax 2002; 57: 226-230.

17 Adams RJ, Smith BJ, Ruffin RE. Factors associated with hospital admissions and repeat emergency department visits for adults with asthma. Thorax 2000; 55: 566-573.
18 Vollmer WM, O'Hollaren M, Ettinger KM, et al. Specialty differences in the management of asthma. A cross-sectional assessment of allergists' patients and generalists' patients in a large HMO. Arch Intern Med 1997; 157: 1201-1208.

19 Kanter LJ, Siegel CJ, Snyder CF, Pelletier EM, Buchner DA, Goss TF. Impact of respiratory symptoms on health-related quality of life and medical resource utilization of patients treated by allergy specialists and primary care providers. Ann Allergy Asthma Immunol 2002; 89: 139-147.

20 Cote J, Cartier A, Robichaud P, et al. Influence on asthma morbidity of asthma education programs based on selfmanagement plans following treatment optimization. Am J Respir Crit Care Med 1997; 155: 1509-1514. 